\title{
Magnetic properties lithium-doped manganite single crystals
}

\author{
S. N. Barilo, G. L. Bychkov, V. I. Gatalskaya, \\ L. A. Kurochkin, and V. P. Sokol \\ Institute of Solid State and Semiconductor Physics of National Academy of Science of Belarus \\ 17 P. Brovki Str., Minsk 220072, Belarus \\ E-mail: bars@ifttp.bas-net.by \\ H. Szymczak, R. Szymczak, and M. Baran \\ Institute of Physics of Polish Academy of Science, 32/46 Al. Lotnikov Str., Warsaw 02-668, Poland \\ E-mail: szymr@ifpan.edu.pl
}

Received October 26, 2000

\begin{abstract}
Magnetic and resistivity properties of $\mathrm{La}_{1-x} \mathrm{Li}_{x} \mathrm{MnO}_{3}$ single crystals with the perovskite structure at lithium concentrations $x=0.06$ and 0.2 are investigated over a broad temperature range at fields of up to $50 \mathrm{kOe}$. The Curie temperature is $T_{C}=90 \mathrm{~K}(x=0.06)$ and $300 \mathrm{~K}(x=0.2)$, respectively. The electrical resistance of the single crystal with $x=0.06$ increases steadily with temperature decrease, while the crystal with $x=0.2$ exhibits a magnetoresistance effect of $\sim 8 \%$ at an external field of $9 \mathrm{kOe}$. The different dependence of the magnetizations $M_{F C}$ and $M_{Z F C}$ of the sample with lower lithium content in the temperature range below $T_{C}$ is associated with the formation of a cluster glass and is caused by high coercivity and magnetic anisotropy of the crystal.
\end{abstract}

PACS: 75.30.Vn, 75.70.Lk, 75.60.Ej

\section{Introduction}

The discovery of colossal magnetoresistance $(\mathrm{CMR})$ in lanthanum manganites in the near-room temperature range has inspired a renewed interest in the study of the magnetic, resistivity, and other properties of the materials described by the formula $\mathrm{Ln}_{1-x} \mathrm{~A}_{x} \mathrm{MnO}_{3}$, where Ln stands for the lanthanide ions and $\mathrm{A}$ is the doping element (see, for example, Refs. 1, 2 for a review). This interest arose both from the possibility of using them as potential materials for the read heads of mass-media devices, magnetic field sensors and magnetic coolers, and from the need to test various models which show a strong correlation either among the transport and magnetic properties or between the crystal structure transition and magnetism, since the CMR phenomenon originates from electron, spin, and elastic interaction of the system. Despite a vast number of papers devoted this problem, the nature of the CMR is still not clearly understood.

The divalent ions $\mathrm{Ca}^{2+}, \mathrm{Sr}^{2+}, \mathrm{Ba}^{2+}$, and $\mathrm{Pb}^{2+}$ are generally used as doping elements. The parent compound $\mathrm{LaMnO}_{3}$ is an antiferromagnetic insulator, whereas the elevated conductivity of the doped crystals is due to holes arising from the substitution of divalents ions for the $\mathrm{La}^{3+}$ ions. Manganese ions in such crystals have mixed valence and high spin state: $\mathrm{Mn}^{3+}\left(t_{2 g}^{3} e_{g}^{1} ; S=2\right)$ and $\mathrm{Mn}^{4+}\left(t_{2 g}^{3} ; S=\right.$ $=3 / 2$ ), and an interaction between them could lead to metallic conductivity and ferromagnetism, let us say, by Zener's double exchange mechanism $[3,4]$. However, the lanthanum ion can be substituted not only by the above divalent ions, but also by other ones, such as, monovalent ions of alkali metals [5-8]. As we know, ceramic samples with $\mathrm{K}^{+}, \mathrm{Li}^{+}$, and $\mathrm{Na}^{+}$concentrations less than $25 \%$ have been hitherto investigated. In Ref. 7 the magnetic and electrical properties of the compound $\mathrm{La}_{1-x} \mathrm{~K}_{x} \mathrm{MnO}_{3}$ $(0.05 \leq x \leq 0.2)$ with the rhombohedral structure were studied. All the samples were ferromagnets and the Curie temperature increased steadily as the potassium content increased. The maximum $T_{C}=308 \mathrm{~K}$ was observed at $x=0.2$. The value of the magnetoresistive effect in these compounds was reported to be comparable to that of the CMR in lanthanum manganites doped with alkaline earth 
ions. The influence of the magnetic field on the entropy of potassium- and sodium-containing lanthanum manganites was also explored [8,9]. In these compounds the $T_{C}$ value increases as the concentration of the doping monovalent ion increases over the interval $0.075 \leq x \leq 0.2$, to reach $334 \mathrm{~K}\left(\right.$ for $\mathrm{Na}^{+}$) and $344 \mathrm{~K}\left(\right.$ for $\mathrm{K}^{+}$), while for $x=0.075$ the $T_{C}$ value was significantly lower than $193 \mathrm{~K}\left(\right.$ for $\mathrm{Na}^{+}$) and $230 \mathrm{~K}$ (for $\mathrm{K}^{+}$), respectively. A distinct change of magnetic entropy of the samples was found in measurements of the field and temperature dependences of the magnetization. The authors ascribe it a the transition from the ferromagnetic to the paramagnetic state and believe that all these materials hold much promise for use as the working substance of magnetic coolers. We are also familiar with two papers in which $\mathrm{La}_{1-x} \mathrm{Li}_{x} \mathrm{MnO}_{3}$ compounds were investigated. In Ref. 10 lithium was intercalated into $\mathrm{LaMnO}_{3}$ either with the rhombohedral or orthorhombic structure by electrochemical reaction. In the first case $T_{c}$ was equal to $216 \mathrm{~K}$ for the sample with $x=0.05$ and to $249 \mathrm{~K}$ at $x=0.07$, and the average magnetic moment per manganese ion varied from 3.35 to $3.33 \mu_{B}$, that is, was in accordance with the increase of the lithium concentration. While in the case of $\mathrm{LaMnO}_{3}$, with the initial orthorhombic structure $\left(T_{C}=131 \mathrm{~K}\right)$, the introduction of lithium in amounts of more than $3 \%$ appeared to be impossible. In this instance $T_{C}=115 \mathrm{~K}$, and the average magnetic moment per manganese ion decreased from 3.14 to $3.11 \mu_{B}$ at $x=0.03$. In Ref. $11 T_{C} \sim 200 \mathrm{~K}$ was obtained for polycrystalline $\mathrm{La}_{0.87} \mathrm{Li}_{0.13} \mathrm{MnO}_{3}$, and the extrapolation of the magnetic moment to zero temperature yielded $3.29 \mu_{B}$ per $\mathrm{Mn}$ ion, which was substantially lower than the value of $3.76 \mu_{B}$ predicted in terms of the double exchange model.

In this paper we present experimental data from studies of the magnetization and electrical conductivity of two $\mathrm{La}_{1-x} \mathrm{Li}_{x} \mathrm{MnO}_{3}$ single crystals with $x=0.06$ and 0.2 , respectively.

\section{Experimental details and samples}

Single crystals of $\mathrm{La}_{1-x} \mathrm{Li}_{x} \mathrm{MnO}_{3}$ were grown using a platinum crucible by spontaneous crystallization from a $\mathrm{La}_{2} \mathrm{O}_{3}-\mathrm{Mn}_{2} \mathrm{O}_{3}-\mathrm{Li}_{2} \mathrm{O}-\mathrm{B}_{2} \mathrm{O}_{3}$ flux melt. Flux melt cooling at a rate of $0.05^{\circ} \mathrm{C} / \mathrm{h}$ over the temperature range from 1250 to $1200^{\circ} \mathrm{C}$ was undertaken to grow crystals of close to cubic shape with an average size up to $3 \mathrm{~mm}$ per side. Powder $\mathrm{x}$-ray diffraction analysis revealed sample 1 (with Li content $x=0.06$ ) to be of an orthorhombically distorted perovskite structure with the crystal lattice parameters: $a=5.485 \AA, b=5.522 \AA$, and $c / \sqrt{2}=5.495 \AA$, whereas sample $2(x=0.2)$ had a nearly cubic.

Measurements of the temperature and field dependences of the magnetization $M(T, H)$ were taken in a field up to $50 \mathrm{kOe}$ over a broad temperature range by means of a SQUID magnetometer (Quantum Design MPMS-5). The samples resisitivity was checked by the standard four-probe technique in a magnetic field of up to $60 \mathrm{kOe}$.

\section{Results}

The temperature dependences of the magnetization of the single crystal with low lithium content (sample 1) measured in a field-cooling regime (FC) or in the zero-field cooling ( $\mathrm{ZFC}$ ) regime are presented in Fig. 1. An abrupt transition to the ferromagnetic state as the temperature decreases occurs at $T_{C}=90 \mathrm{~K}$. At temperatures below $T_{C}$ a sharp distinction is observed between the curves $M_{F C}(T)$ and $M_{Z F C}(T)$. In particular, the magnetization $M_{F C}$ increases at $T<T_{C}$ to the lowest temperature, while, in contrast, a broad maximum (cusp) at $T_{f}=80 \mathrm{~K}$ is noted in the $M_{Z F C}(T)$ dependence. At the residual SQUID field $H \sim 2$ Oe the curves $M_{F C}(T)$ and $M_{Z F C}(T)$ match, beginning with $T=T_{f}$, as temperature rises. With increasing magnetic field (Fig. 2) the difference in the $M_{F C}(T)$ and $M_{Z F C}(T)$ dependences becomes less pronounced: the cusp on the $M_{Z F C}$ curve broadens, and $T_{f}$ shifts to lower temperatures. Finally, in a field of $6 \mathrm{kOe}$ the difference between $M_{F C}(T)$ and $M_{Z F C}(T)$ practically disappears.

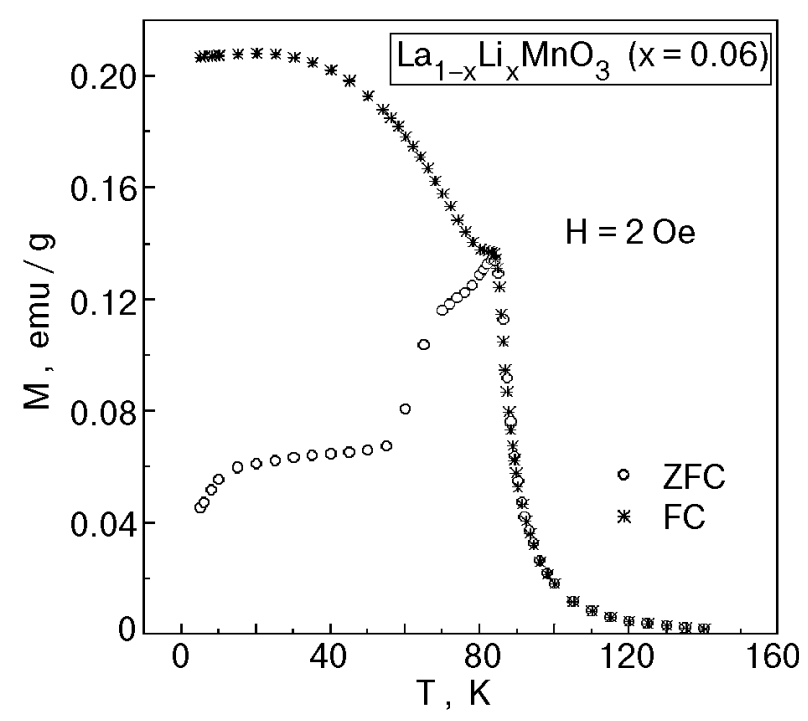

Fig. 1. Temperature dependence of the magnetization $M_{F C}$ (*) and $M_{Z F C}(\bigcirc)$ at $H=2$ Oe for sample 1 . 


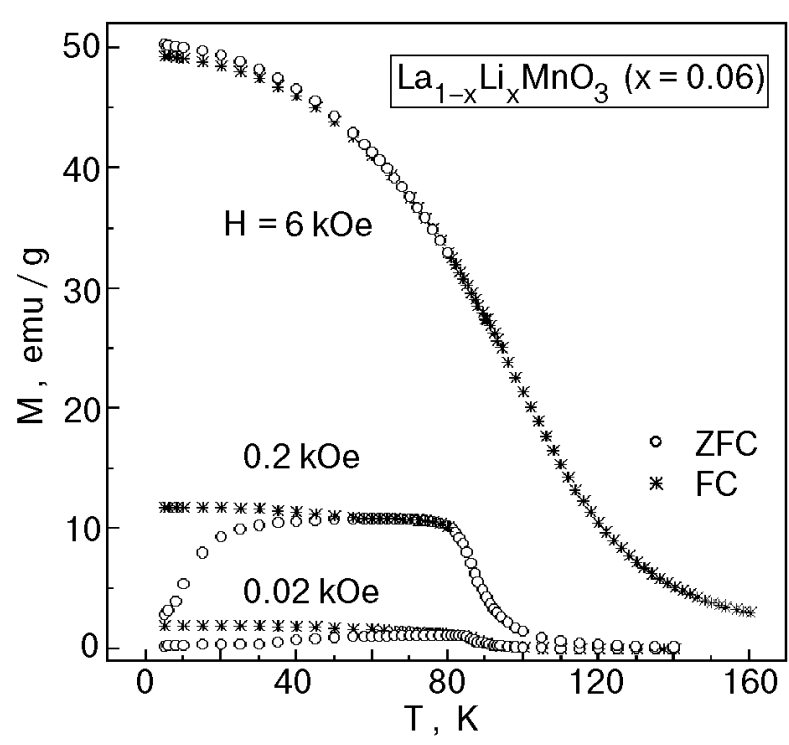

Fig. 2. Temperature dependence of the magnetization $M_{F C}$ (*) and $M_{Z F C}(\bigcirc)$ in various magnetic fields for sample 1 .

Magnetization isotherms of sample 1 are shown in Fig. 3. It is seen that at low temperatures the magnetization does not reach saturation in a field of $50 \mathrm{kOe}$ and that hysteresis is observed in fields of up to $4 \mathrm{kOe}$ (see Fig. 3 inset). The temperature dependence of the magnetization $M(T)$ of sample 1 in a field of $50 \mathrm{kOe}$ is shown in Fig. 4. In this case the average magnetic moment per $\mathrm{Mn}$ ion amounts to $2.6 \mu_{B}$. Note that the field and temperature dependences of the magnetization for different crystallographic directions of the applied magnetic field are practically identical.

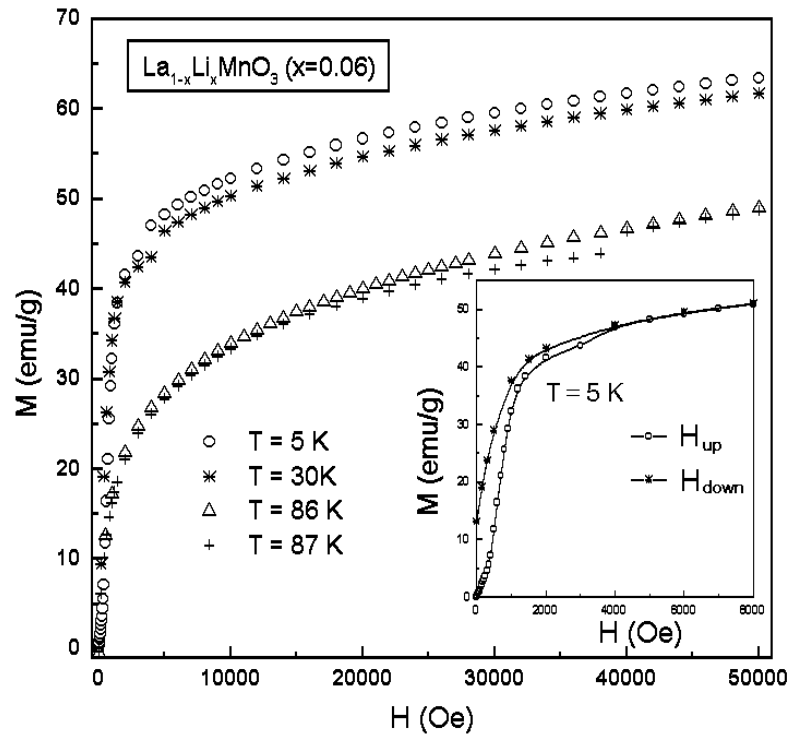

Fig. 3. Magnetization isotherms for sample 1. Inset: $M(H)$ dependence at $5 \mathrm{~K}$ in the low field range.

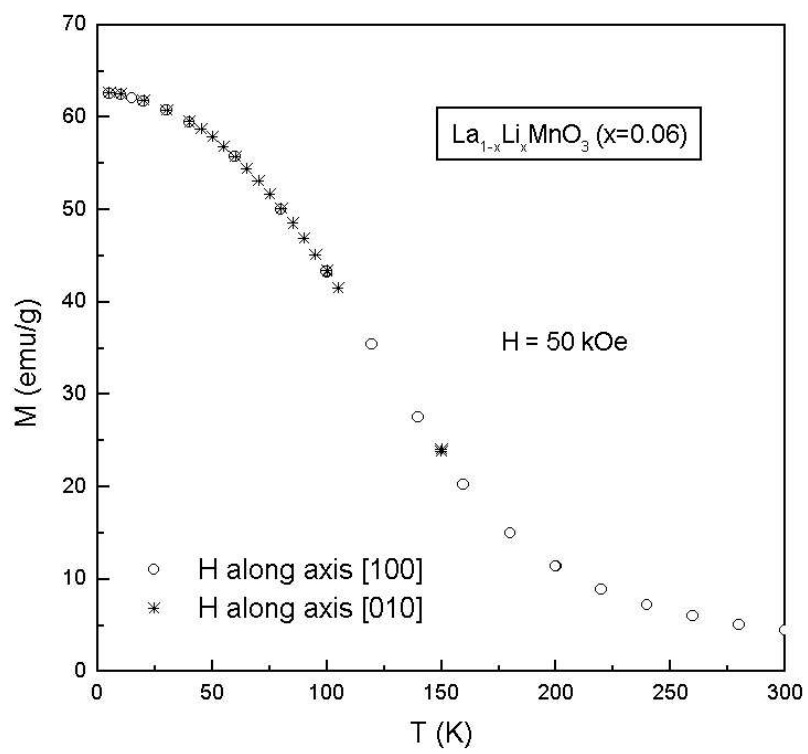

Fig. 4. Temperature dependence of the magnetization $M(T)$ at a field of $50 \mathrm{kOe}$ applied in two different crystal directions for sample 1.

The temperature dependences $M_{F C}(T)$ and $M_{Z F C}(T)$ for the single crystal of high lithium content (sample 2) at various directions of the applied field are given in Fig. 5. One can see that the transition to the ferromagnetic state takes place at room temperature $T_{C}=300 \mathrm{~K}$. Figure 5 calls attention to the fact that the difference between $M_{F C}(T)$ and $M_{Z F C}(T)$ in all directions of the applied magnetic field is small. In particular, $M_{F C}$ remains practically temperature-independent below $T_{C}$, and $M_{Z F C}$ reveals a weak decrease down to the lowest temperature. The field dependences $M(H)$ at

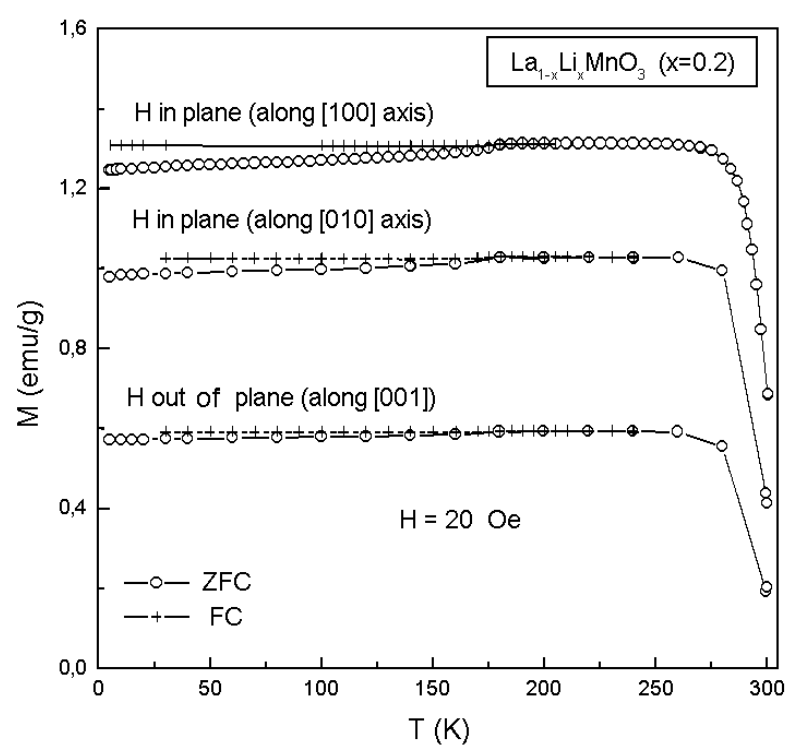

Fig. 5. Temperature dependences of the magnetization $M_{F C}(T)$ and $M_{Z F C}(T)$ in fields of various orientations for sample 2 . 


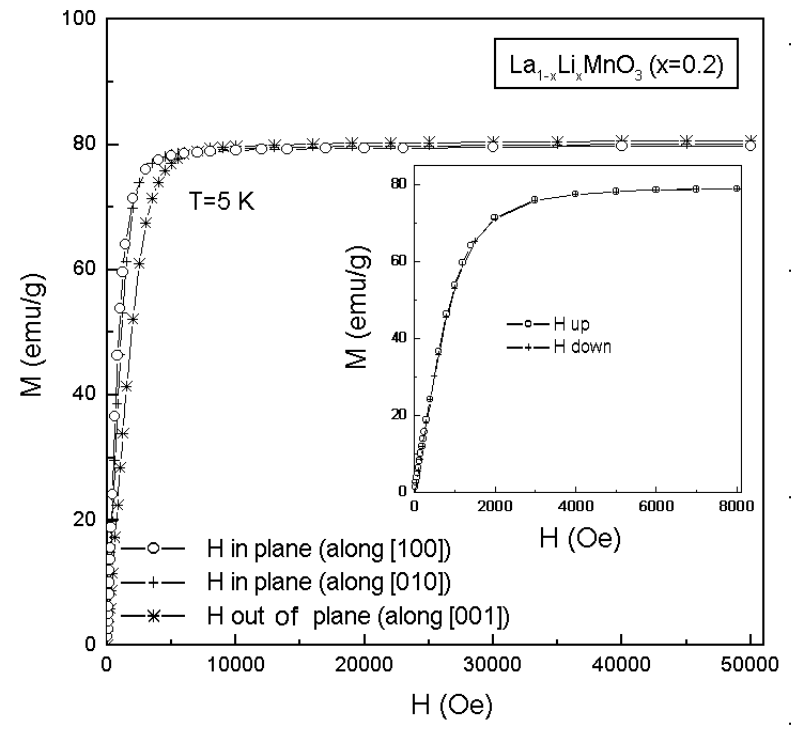

Fig. 6. Field dependences of the magnetization at $5 \mathrm{~K}$ for various field orientations for sample 2. Inset: $M(H)$ dependence in the low-field range.

$5 \mathrm{~K}$ for sample 2 (Fig. 6) showed that the magnetization achieved saturation for all directions of the applied magnetic field, even in fields of 3-4 kOe. The low-field portion of the magnetization given in the inset of Fig. 6 displays practically reversible behavior of the dependence $M(H)$. The temperature dependence of magnetization in a field of $50 \mathrm{kOe}$ (Fig. 7) characterizes the magnetic anisotropy of the sample 2.

Preliminary measurements of the dependence of the electrical resistance on temperature for both the single crystals (Figs. 8, 9) showed a dramatic in-

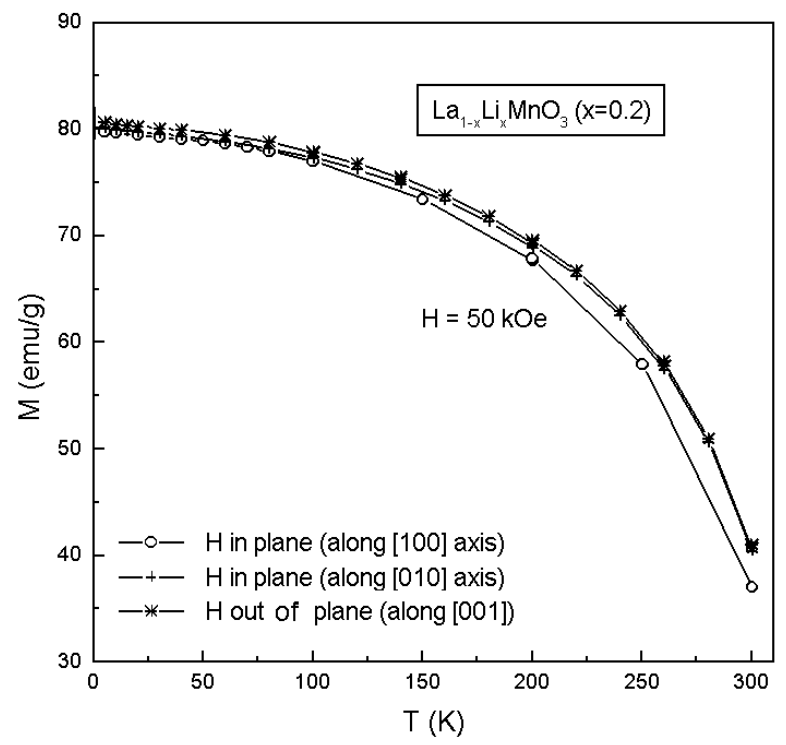

Fig. 7. Temperature dependences of the magnetization in a field $H=50 \mathrm{kOe}$ of various orientations for sample 2 .

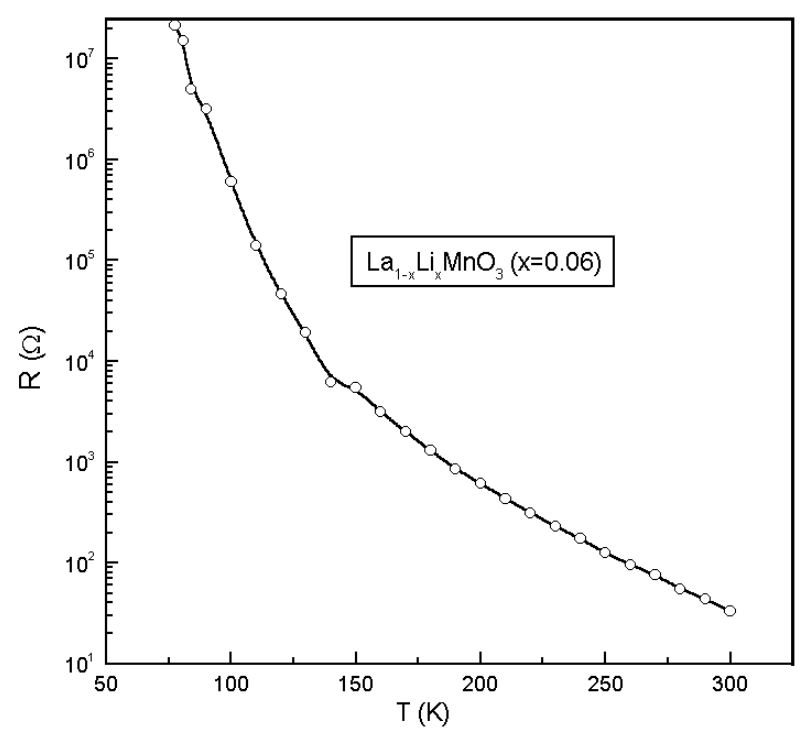

Fig. 8. Temperature dependence of the electrical resistivity for sample 1 .

crease the resistance of sample 1 as the temperature is lowered from 300 to $77 \mathrm{~K}$. The typical rise in the resistance by some orders of magnitude continues to magnetic fields of up to $50 \mathrm{kOe}$. The resistivity of sample 2 rises with increasing temperature in the range from 77 to $300 \mathrm{~K}$, and thereafter its decrease is observed. The resistivity of sample 2 decreased when the magnetic field was applied, while the metal-insulator transition temperature, accompanied by a resistivity maximum, increased from $\sim 300 \mathrm{~K}$ (at zero field) to $315 \mathrm{~K}$ at the maximum available field of $9 \mathrm{kOe}$. The highest variation of the resistance was noted at temperatures near

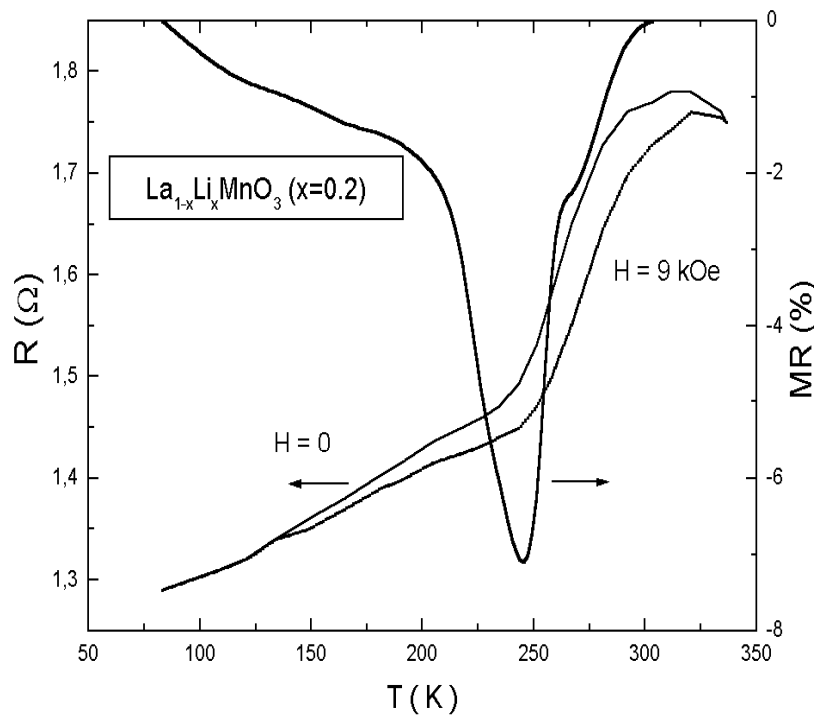

Fig. 9. Temperature dependence of the electrical resistivity in an external field $H=9 \mathrm{kOe}$ or in the absence of field, and also the temperature dependence of the magnetoresistance for sample 2 . 
$250 \mathrm{~K}$. In this case the CMR effect value amounted to $8 \%$.

\section{Discussion of experimental data}

The magnetization and resistivity of two single crystals of lanthanum lithium manganites with different lithium content were studied. The transition from the paramagnetic to the ferromagnetic state on decreasing temperature occurs at $90 \mathrm{~K}$ (sample 1) and at $300 \mathrm{~K}$ (sample 2), respectively. The measurements of magnetization versus magnetic field dependences of sample 1 exhibited a close to isotropic $M(H)$ behavior and the same magnitude of the spontaneous magnetization for various directions of the applied magnetic field. The magnetization of the crystal does not achieve saturation in a field of up to $50 \mathrm{kOe}$ at a temperature of $5 \mathrm{~K}$. In contrast, the magnetization of sample 2 at the same temperature achieves saturation at an external field of 3-4 kOe, depending on the crystal orientation. The saturated magnetization $\sim 80 \mathrm{emu} / \mathrm{g}$ of sample 2 in the field of $50 \mathrm{kOe}$ at $5 \mathrm{~K}$ corresponds to an average magnetic moment per Mn ion of $3.11 \mu_{B}$. Based on the following cation distribution

$$
\left(\mathrm{La}_{1-x}^{3+} \mathrm{Li}_{x}^{+}\right)_{A}\left(\mathrm{Mn}_{1-2 x}^{3+} \mathrm{Mn}_{2 x}^{4+}\right)_{B} \mathrm{O}_{3}
$$

the magnetic moment per manganese ion was estimated using the Zener's model of double exchange of the $\mathrm{Mn}^{3+}-\mathrm{Mn}^{4+}$ pairs in the $B$ sublattice of the perovskite structure. Then, assuming a stoichiometric oxygen concentration, one can get the following calculated value of the saturated magnetic moment:

$$
\mu_{s \text { calc }}=(1-2 x) \mu_{s}\left(\mathrm{Mn}^{3+}\right)+2 x \mu_{s}\left(\mathrm{Mn}^{4+}\right)
$$

for complete spin ordering $\mu_{s_{3} \text { calc }}=g S$, where $g=2$ and $S=2$ or $3 / 2$ for $\mathrm{Mn}^{3+}$ and $\mathrm{Mn}^{4+}$, respectively. Because the calculated moment value $\mu_{s \text { calc }}=3.6 \mu_{B}$ for sample 2 ( $\mathrm{Li}$ content $x=0.2$ ) exceeds somewhat the experimental value we suggest that this may be related to some disorder, such as oxygen nonstoichiometry in the crystal or partial substitution of manganese sites by lithium. Similar calculations for sample 1 yields the value $\mu_{s \text { calc }}=3.12 \mu_{B}$, which is also much higher than the experimental magnitude of $2.6 \mu_{B}$ (Figs. 3 and 4). The difference between the two single crystals is most pronounced in the $M_{F C}(T)$ and $M_{Z F C}(T)$ behavior in low applied fields. For instance, at a magnetic field in the interval 2-200 Oe a clearly defined cusp is observed on the $M_{Z F C}(T)$ dependence of sample 1 in the temperature range below $T_{C}$, whereas for sample $2 M_{Z F C}$ decreases slightly as the temperature is lowered (Figs. 1, 2, and 5). Distinct behavior of the $M_{F C}(T)$ and $M_{Z F C}(T)$ curves in ordered magnetic systems was also reported in Refs. 12-15. In particular, the properties of $\mathrm{LaMnO}_{3+\delta}$ at low temperature are interpreted in terms of successive phase transitions, that is, as the temperature decreases, first ferromagnetic ordering in weakly coupled clusters occurs in the system and then the cluster-glass phase develops. A distinction between the $M_{F C}$ and $M_{Z F C}$ behavior in a temperature range close to $T_{C}$ is peculiar just to the cluster-glass state. Of course this distinction disappears as the magnetic field increases. We believe that in sample 1 an analogous cluster-glass state becomes apparent. It is known that the formation of ferromagnetic clusters accompanied by a strong charge localization results in the appearance of the ferromagnetic insulator state. The latter was observed in sample 1 (Fig. 8). In Refs. 14, 15 the mechanism of the cluster-glass formation in the magnetic compounds of $\mathrm{La}_{0.5} \mathrm{Sr}_{0.5} \mathrm{CoO}_{3}, \mathrm{SrRuO}_{3}$, $\mathrm{La}_{0.7} \mathrm{Ca}_{0.3} \mathrm{MnO}$, and $\mathrm{La}_{0.5} \mathrm{Sr}_{0.5} \mathrm{MnO}_{3}$ was discussed. The difference between the temperature dependences of $M_{F C}$ and $M_{Z F C}$ as well as the variation of the cusp temperature on the $M_{Z F C}(T)$ curve as the external field increases and the temperature dependence of the magnetic susceptibility in low fields are specified by the coercive field and its variation with temperature $H_{c}(T)$. Specifically, the ratio of the applied magnetic field $H$ to $H_{c}$ measures the magnetocrystalline contribution to the magnetic anisotropy. To put it differently, the magnitude and temperature dependence of the coercive field play a significant role in the magnetization of the compound at a given value of the external magnetic field. Using the above system as an example, it is shown the field-cooled susceptibility of the sample $\chi_{F C}=M_{F C} / H$ or the zero-field-cooled susceptibility $\chi_{Z F C}=M_{Z F C} / H$ does not differ, provided that the applied magnetic field is much higher than the coercive field [14,15]. At the same time, this distinction at low magnetic field could not be neglected for a highly coercive material $\left(H_{c}>>H\right)$. The coercive field of sample 1 is high $\left(H_{c} \sim 1 \mathrm{kOe}\right)$ at $5 \mathrm{~K}$ and is practically absent $\left(H_{c} \sim 2-3 \mathrm{Oe}\right)$ at $30 \mathrm{~K}$. Therefore at magnetic fields ranging from 2 to 200 Oe there is a marked difference in $M_{F C}(T)$ and $M_{Z F C}(T)$, which disappears effectively at $H>1$ kOe. At the same time sample 2 has no hysteresis (Fig. 6, inset), while the temperature dependences $M_{F C}(T)$ and $M_{Z F C}(T)$ differ slightly (Fig. 5). In turn, the transition of this sample to the ferromagnetic state at near-room temperature is 
accompanied by a pronounced magnetoresistive effect $(\mathrm{CMR} \sim 8 \%$ at a field of $9 \mathrm{kOe})$.

\section{Conclusions}

Single crystals of $\mathrm{La}_{1-x} \mathrm{Li}_{x} \mathrm{MnO}_{3}$ at $x=0.06$ and $x=0.2$ were first grown from the flux melt with the structure of orthorhombically distorted (sample 1) and practically cubic (sample 2) perovskite. Both the crystals revealed the ferromagnetic ordering at $T_{C}=90$ and $300 \mathrm{~K}$, respectively. The electrical resistance of sample 1 increases steadily as the temperature decreases, whereas the resistivity of sample 2 drops abruptly at temperatures below $T_{C}$. Preliminary magnetoresistance measurements showed the CMR effect of $\sim 8 \%$ at a field of $9 \mathrm{kOe}$ only for sample 2 . The different temperature dependence of the magnetization at low external field for samples cooled in the field $M_{F C}$ and in its absence $M_{Z F C}$ as well as the appearance of the cluster glass state in sample 1 at $T<T_{C}$ are due to a distinction in the coercivity and magnetic anisotropy. Specifically sample 1 has a high coercive force $H_{c} \sim 1 \mathrm{kOe}$ at $5 \mathrm{~K}$ and a larger magnetic anisotropy than that of sample 2 , which has a negligibly small coercive field $H_{c} \sim 2-3$ Oe at the same temperature.

\section{Acknowledgments}

One of us (VIG) would like to thank National Academy of Sciences of Belarus for financial support during her stay in Warsaw.

1. E. L. Nagaev, Usp. Fiz. Nauk 166, 833 (1996) (in Russian).

2. A. P. Ramirez, J. Phys.: Condens. Matter 9, 8171 (1997).

3. C. Zener, Phys. Rev. 82, 403 (1951).

4. P. G. de Gennes, Phys. Rev. 118, 141 (1960).

5. M. K. Gubkin, T. M. Perekalina, A. V. Bykov, and V. A. Chubarenko, Fiz. Tverd. Tela 35, 1443 (1993) (in Russian).

6. M. Itoh, T. Shimura, J. D. Yu, T. Hayashi, and Y. Inaguma, Phys. Rev. B52, 12522 (1995).

7. C. Boudaya, L. Larossi, E. Dhahri, J. C. Joubert, and A. Cheikh-Rouhou, J. Phys.: Condens. Matter 10, 7485 (1998).

8. W. Zhong, W. Chen, W. P. Ding, N. Zhang, Y. W. Du, and Q. I. Yan, Solid State Commun. 106, 55 (1998).

9. W. Zhong, W. Chen, W. P. Ding, N. Zhang, A. Hu, Y. W. Du, and Q. I. Yan, J. Magn. Magn. Mater. 195, 112 (1999).

10. M. Itoh, T. Shimura, T. Hayashi, and Y. Inaguma, Solid State Communs. 97, 179 (1996).

11. Sh. Nakamura, K. Nanba, and Sh. Iida, J. Magn. Magn. Mater. 177-181, 884 (1998).

12. C. Ritter, M. R. Ibarra, J. M. De Teresa, P. A. Algazabel, C. Marquina, J. Blasko, J. Garcia, S. Ozeroff, and S. W. Cheong, Phys. Rev. B56, 8902 (1997).

13. P. A. Joy, P. S. A. Kumar, and S. K. Date, J. Phys.: Condens. Matter 10, 11049 (1998).

14. P. S. A. Kumar, P. A. Joy, and S. K. Date, J. Phys.: Condens. Matter 10, L487 (1998).

15. L. Ghivelder, I. A. Castillo, M. A. Gusmao, J. A. Alonso, and L. F. Cohen, Phys. Rev. B60, 19184 (1999). 